\title{
A PHOTOELECTRIC METHOD FOR THE QUANTITATIVE DETERMINATION OF ERYTHROCYTE FRAGILITY
}

\author{
BY FRANCIS T. HUNTER \\ (From the Laboratory of the Baker Memorial, Massachusetts General Hospital, Boston)
}

(Received for publication April 27, 1940)

The method described in most textbooks for the determination of the fragility range of erythrocytes possesses several disadvantages. In the first place, quantitation is not possible because unmeasured drops of blood are placed in tubes containing only roughly estimated amounts of hypotonic salt solution. As might be expected, a semi-quantitative technique of this type, when applied to clinical investigation, gives grossly irregular fragility curves such as those obtained by Vaughan (1). Secondly, since the observed degree of hemolysis in any one tube is dependent upon diffusion of liberated oxyhemoglobin through the liquid above the sedimented cells, other factors such as temperature, the anticoagulant used, the amount of bile pigments present in the plasma, and the presence of hemolytic organisms, may affect the results erroneously. It seemed desirable, therefore, to devise for this test a technique rapid and simple enough for clinical application, and at the same time sufficiently accurate for purposes of research.

Because of the fact that the amount of oxyhemoglobin liberated by a hypotonic salt solution is a direct function of the number of cells hemolyzed; it is obvious that a determination of the freed oxyhemoglobin resulting from such partial hemolysis may be used as a hemolytic index. Furthermore, as complete hemolysis results from laking erythrocytes in a 0.10 per cent solution of sodium carbonate (2), it is possible to express the hemolysis occurring in a given strength of salt solution in terms of total hemolysis. Thus, the quantitative determination of partial hemolysis in any strength of salt solution can be easily made if a measured amount of blood is placed in a centrifuge tube containing a definite quantity of the salt solution and, after partial hemolysis has been completed, the unhemolyzed cells are centrifuged off and the oxyhemoglobin in solution in the supernatant fluid estimated by means of a photoelectric colorimeter. Since the technique of the latter instrument is well established (2), it need not be outlined here.

\section{METHOD}

Place 1 cc. of a filtered 1.6 per cent solution of sodium oxalate in a test tube. Boil over an open flame until all water and vapor have been driven off. With a clean dry syringe and needle obtain exactly $5 \mathrm{cc}$. of blood from an arm vein and transfer quickly to the prepared test tube. Mix at once to prevent clotting.

For the determination of partial hemolysis, prepare twenty-six stock solutions of sodium chloride decreasing its strength by 0.02 per cent from 0.70 per cent to 0.20 per cent, inclusive. These must be accurately made and must be kept in well-stoppered bottles. Into one of twenty-six ${ }^{1}$ clean centrifuge tubes transfer exactly 10 cc. of the 0.70 per cent salt solution and label; and in 2 like manner place $10 \mathrm{cc}$. of the other salt solutions in the remaining tubes. With a volumetric pipette transfer exactly $0.05 \mathrm{cc}$. of the well-mixed oxalated blood to each tube, place a rubber cap on each, and invert several times to obtain complete mixing. Allow to stand at room temperature for at least thirty minutes, then invert each tube again, place in a centrifuge, and spin at moderate speed (1500 r.p.m.) for fifteen minutes. Remove the tubes from the centrifuge, carefully decant the supernatant fluid from each, and transfer separately to clean photoelectric absorption cells. Using an appropriate color filter, make determinations of the light transmission, and read off the grams of oxyhemoglobin from a previously prepared photoelectric conversion graph.

To obtain complete hemolysis, place $10 \mathrm{cc}$. of a 0.1 per cent solution of sodium carbonate in 2 test tube and transfer to it exactly $0.05 \mathrm{cc}$. of the same blood and mix immediately. Fill a photoelectric absorption cell with this solution, and in a similar manner determine the grams of oxyhemoglobin present. If the latter figure is low, more accurate results may be obtained by using 0.1 cc. of blood both in the sodium carbonate and in the hypotonic salt solution tubes.

Calculation. Divide the number of grams of oxyhemoglobin resulting from hemolysis in each centrifuge tube by the number of grams obtained from complete laking with sodium carbonate. These figures represent the per cent of total hemolysis occurring in each strength of salt solution.

Plotting of curves. On rectangular coordinate paper mark off the salt solutions in order of decreasing strength along the abcissa, and the per cent hemolysis in increasing order along the ordinate. Plot the per cent hemoly-

1 In respect to a particular blood, if some idea is obtained beforehand of where hemolysis is apt to begin, the number of solutions may be reduced in number. 
sis for each salt solution and connect the points in a continuous curve. If the technique has been carefully carried out, the curve will ordinarily be sigmoid in character-that is, convex toward the base at lower percentages, and convex upward as hemolysis nears completion. In some instances, particularly in the case of bloods showing increased fragility, the curve may show more than one maximal slope. The significance of this occasional variation from the usual curve, however, is by no means clear and requires further investigation.

\section{DISCUSSION}

Sigmoid curves such as these represent the first integral of curves of the probability type; or, in other words, the plotted first differential of such sigmoid curves gives the probability-distribution of the erythrocyte fragility of any particular blood for varying strengths of salt solution. As has been shown by Ponder (3), when the per cent of total hemolysis is plotted against time, various hemolytic agents give typical sigmoid curves. Moreover, if one plots Ponder's values for the per cent hemolysis produced by varying concentrations of a lysin at a given time interval after lysis has begun, it can be demonstrated that each of the resulting curves is also sigmoid in character. One can affirm, therefore, that hypotonic solutions behave in this respect like other lysins.

The curve of normal hemolysis in Figure 1 was derived from fragility determinations of the erythrocytes of twelve normal individuals. The per cent hemolysis for each strength of salt solu- tion was first averaged for the twelve bloods, and these averages were then plotted on arithmetic probability paper (Figure 2) (4). In general, the points fell on a straight diagonal line, and those not so doing exhibited only small deviations to either side. Thus, after taking from the probability coordinate corrected points for the per cent hemolysis for the salt concentrations in question, the normal curve was constructed on rectangular coordinates as shown.

It will be seen that normal blood shows less than 2 per cent hemolysis in 0.50 per cent salt solution and reaches a maximal hemolysis at $\mathbf{0 . 3 4}$ per cent. If every blood showed an equal deviation from the mean fragility, all curves, of course, would be parallel to each other regardless of their position in respect to the abcissa. That this does not occur is obvious, and hence it is impossible to construct a whole curve from the determination of the per cent hemolysis produced by a single concentration of salt. Nevertheless, by using only three appropriate strengths of salt (viz. 0.52 , $0.42,0.32$ per cent), the position of any curve can be approximated with minimal effort, and for most clinical purposes this should suffice.

In Figure 1 the curve beginning at high salt concentrations represents a case of hemolytic jaundice, while the two lying to the right of the normal curve exemplify the fragility range of a case of Cooley's anemia and of a case of chronic benzol poisoning. Curves have also been ob-

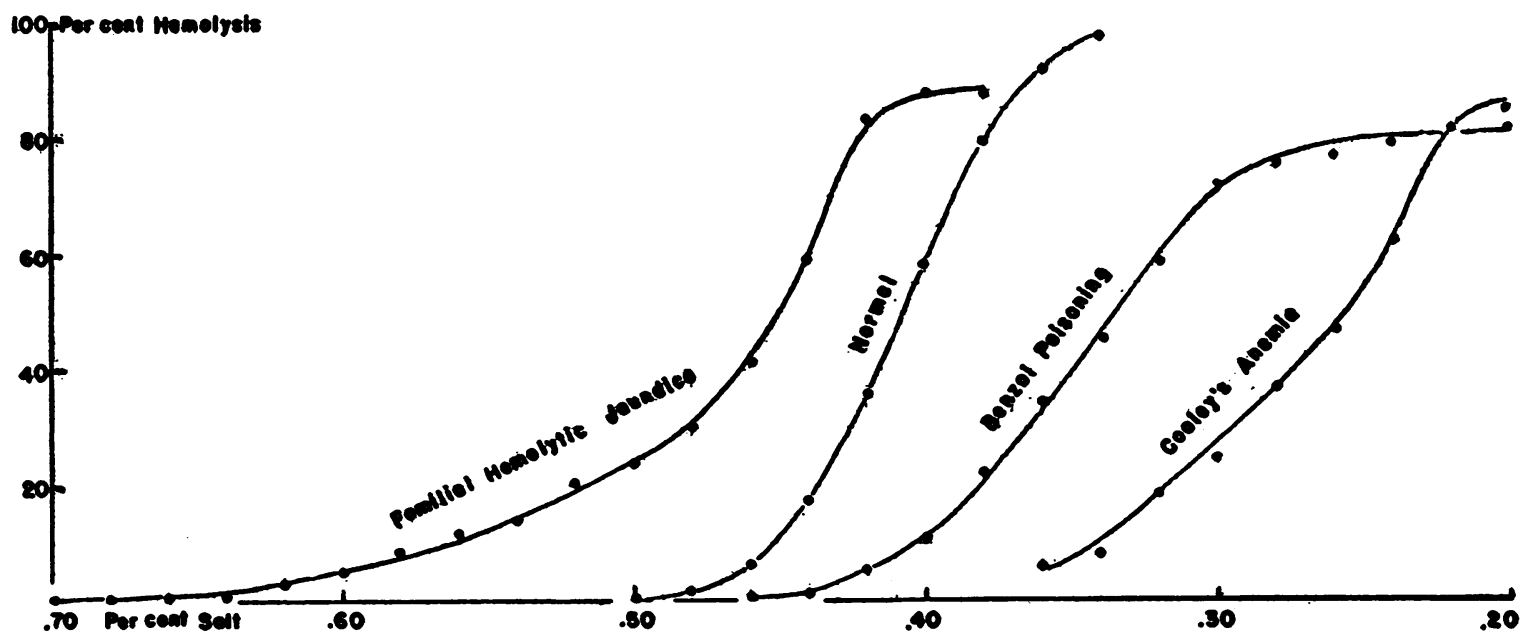

Fig. 1. Per Cent Hryolysis Plotred Against Strength of Salt Solution 


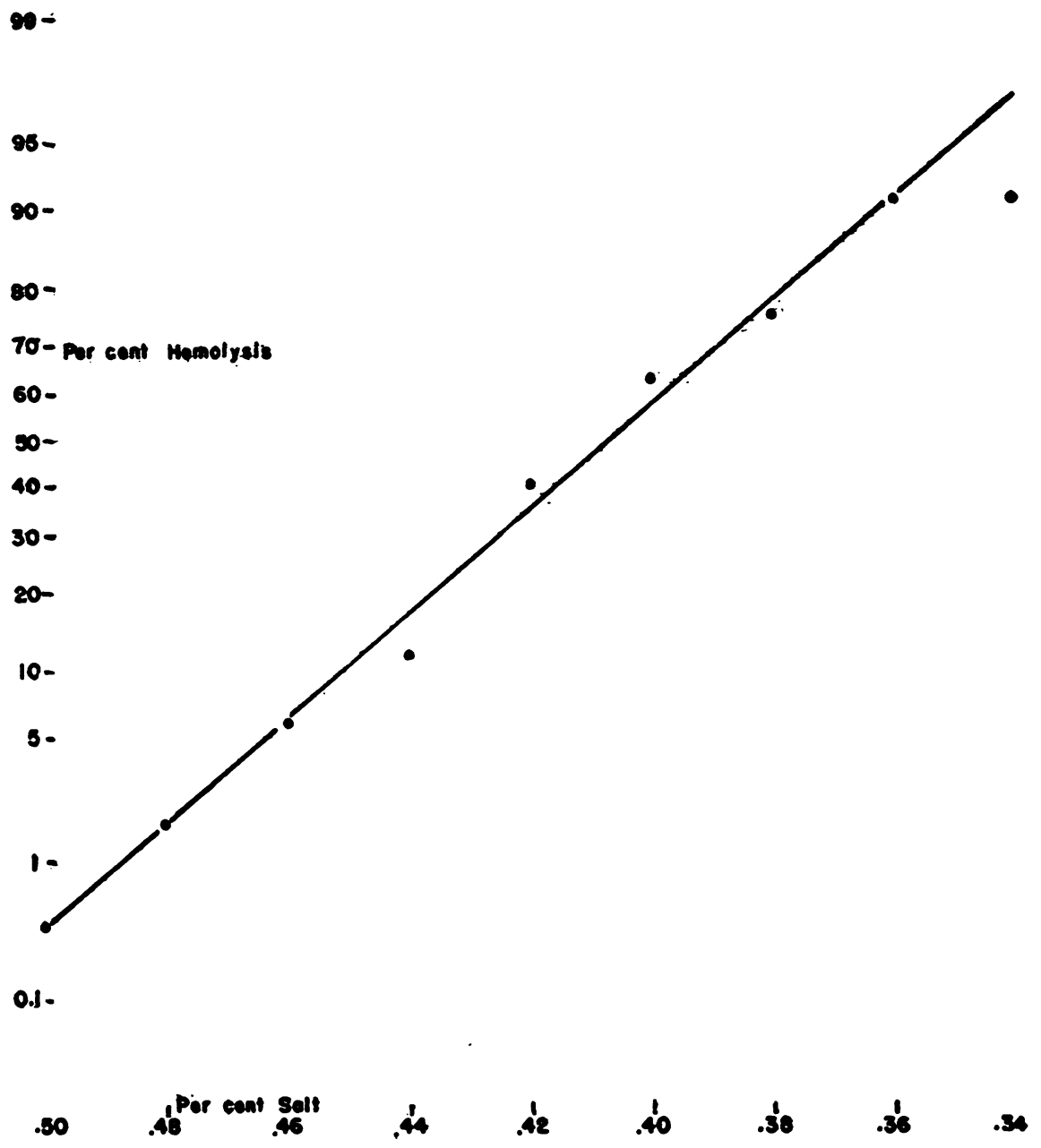

Fig. 2. Per Cent Henolysis Plotted on Probability Paper Against Strength of Salt Solution

These points represent the average hemolysis in 12 normal individuals for varying salt strengths.

tained from additional cases of hemolytic jaundice and from various other disorders of the hematopoietic organs but, since these showed the same general pattern, it did not seem worth while to reproduce them here.

For clinical purposes it has been found that increased fragility can be easily ruled out by placing $0.05 \mathrm{cc}$. of blood in $10 \mathrm{cc}$. of 0.50 or 0.52 per cent salt solution and centrifuging; if the supernatant fluid shows no evidence of hemolysis to the naked eye, the fragility is normal or less than normal. It has also been observed that, due to the high dilution of blood $(1: 200)$ here employed, when the total oxyhemoglobin of the whole blood is near normal the presence of an icteric index even as high as 100 raises the oxyhemoglobin values by only 4 or 5 per cent. But, when the oxyhemoglobin content of the blood is markedly decreased, the presence of any considerable degree of jaundice introduces an error sufficient at times to warrant replacing the plasma with 0.85 per cent salt solution before the test for fragility is undertaken.

\section{SUMMARY}

1. A photoelectric method for estimating erythrocyte fragility is described. 
2. The sigmoid curves obtained by this technique represent the first integral of the probabilitydistribution of fragility in respect to concentration of salt.

\section{BIBLIOGRAPHY}

1. Vaughan, J. M., Red cell characteristics in acholuric jaundice. J. Path. and Bact., 1937, 45, 561.
2. Sanford, A. H., Sheard, C., and Osterberg, A. E., The photelometer and its use in the clinical laboratory. Am. J. Clin. Path., 1933, 3, 405.

3. Ponder, E., The mammalian red cell and the properties of hemolytic systems. Borntraeger. Berlin, 1934, p. 164.

4. Bell, J. R., Thomas, F. K., and Means, J. H., Studies on red cell diameter. I. In health and in pernicious anemia J. Clin. Invest., 1926, 3, 229. 